\title{
La Suisse urbaine à l'orée du 3e millénaire: risques et défis d'un changement non programmé
}

\begin{abstract}
Résumé
Introduisant, à titre d'éditeur invité, les autres travaux de ce numéro consacré à une présentation de certains aspects des connaissances et pratiques de la géographie humaine en Suisse romande à travers une réflexion prospective sur les devenirs de la Suisse, l'auteur focalise son attention sur les sources du changement liées à la dynamique des structures urbaines, au triple plan de leurs dimensions économiques, sociales et culturelles et sous les deux facettes du développement des entités urbaines et métropolitaines et de la vie en leur sein. Les transformations actuelles, comme celles qui sont les plus probables à court et moyen terme, imposent une remise en question de notre idée de ville et des modèles traditionnels de la géographie urbaine comme aussi de la hiérarchie de ses préoccupations. A ces conditions la géographie pourra, peut-être, contribuer à la lutte, difficile, pour défendre ou refaire l'unité de la ville, serait-ce à une autre échelle.
\end{abstract}

\section{Zusammenfassung}

Diese Nummer ist der Humangeographie in der Westschweiz gewidmet, ihren verschiedenen Aspekten im Hinblick auf die Zukunft unseres Landes. Als Einleitung zu den übrigen Artikeln dieses Heftes legt der Autor sein Schwergewicht auf die Ursachen von Veränderungen, die sich aus der Dynamik der städtischen Strukturen ergeben: auf der wirtschaftlichen, sozialen und kulturellen Ebene sowie unter den beiden Blickwinkeln des Begriffes Stadt und dem ihrer Bewohner und Bewohnerinnen.

Die aktuellen Veränderungen wie auch jene, die kurz- und mittelfristig am wahrscheinlichsten erscheinen, drängen uns, bisherige Vorstellungen der Stadt wie auch die traditionellen Modelle der Stadtgeographen mit ihrer Rangordnung der Fragestellung aufzugeben.

Unter diesen Bedingungen könnte die Geographie einen Beitrag an die schwierige Aufgabe leisten, die Integrität der Stadt entweder zu verteidigen oder nach anderen Mustern neu aufzubauen.

A l'heure où, en dépit de la crise qui frappe l'ensemble des pays industrialisés, les données et les analystes s'accordent encore à conclure qu'aujourd'hui l'«on vit relativement mieux en Suisse que partout ailleurs ou presque» (LAMBELET, 1994) et que sans doute il en sera de même d'ici dix ans (PROGNOS, 1994), que pouvons-nous penser de la Suisse de demain? La géographie peut-elle tenir, sur ce thème, un discours un tant soit peu significatif? Dans la mouvance problématique créée par le vote du 6 dé- cembre 1992, les éditeurs de Geographica Helvetica ont souhaité une prise de parole des géographes romands. Certains d'entre eux ont ainsi trouvé l'occasion d'enrichir leurs travaux en cours d'une dimension prospective, certes bien incertaine, mais qui a ici le mérite d'être ancrée dans la saisie la plus rigoureuse possible des faits actuels. Le principe directeur des articles présentés est bien, selon un mot déjà ancien du fondateur de l'Institut de recherches sur le futur de Vienne: Le futur a déjà commencé.

Dans un monde où l'urbain, c'est une réalité statistique et une expérience quotidienne, devient l'horizon indépassable de notre temps, où chacun reconnait que tout en étant de moins en moins lié à la ville au sens strict, il a «Saisi» (PIVETEAU, 1989) la société toute entière, quel nouvel ordre territorial se profile-t-il pour notre pays? Quels sont les principaux enjeux de l'évolution de nos cadres de vie, en regard d'un idéal démocratique et d'une solidarité sociale et territoriale dont les significations concrètes risquent d'être sérieusement remises en question? Chacun à sa manière, l'un en se fondant sur la modélisation des $d y$ namismes démographiques, l'autre sur l'analyse multivariée de l'évolution du système productif associè au système urbain helvétique, C. HuSsY et A. CUNHA cherchent des réponses, en s'appuyant sur d'importants travaux personnels qui dessinent, pour la première fois peut-être avec cette cohérence, les paysages statistiques suisses. Appuyés sur des référentiels théoriques heureusement explicités, ceux-ci nous paraissent témoigner avec beaucoup d'éloquence, par-delà les chiffres et les cartes, des dynamiques territoriales et sociales helvétiques. E. PIGUET s'intéresse plus précisément à certains aspects de ce qui se profile comme l'un des médiateurs incontournable de notre devenir collectif: l'espace de l'immigration, «les» espaces, précise-t-il justement, en montrant le caractère socialement construit des phénomènes d'intégration et de ségrégation en cours d'actualisation. Comment douter, en prenant connaissance de cet ensemble de données en devenir, que les changements de territorialité que nous sommes en train de vivre débouchent inévitablement sur la prise de conscience qu'il y aura des choix à faire si nous voulons maitriser les changements qui se

Jean-Bernard Racine, Prof., Université de Lausanne, IGUL et HEC, 1015 Lausanne 
dessinent? Et que ces choix dépendront lourdement des transitions structurelles et de style de vie que nous connaitront, mais aussi, et lourdement, de nos représentations et du sens que nous donnerons aux discontinuités dans lesquelles s'inscrit notre relation à l'altérité sociale et environnementale?

A cet égard, et dans un univers de plus en plus marqué par la pluralité de toutes choses, la réflexion de L. Bridel sur le futur des paysages suisses pose une question dont personne ne doutera qu'elle est de portée très générale dans ce pays: «Sommes-nous atteints de schizophrénie?» En conclusion, C. RAFFESTIN se contente de parler de "paradoxes». Mais rien ne parait plus urgent pourtant que de prendre conscience du diagnostic dont ils sont porteurs. Lon sera particulièrement reconnaissant à l'«œil géographique» de notre collègue genevois, qui a le plus explicitement souhaité, et accepté de jouer le jeu de la question "La Suisse dans vingt ans", d'avoir posé, en les révélant et les mettant en scène, les jalons d'une possible guérison de nos si dangereuses discordances, qui transcendent sans aucun doute les oppositions révélées le 6 décembre 1992 entre Romands et Alémaniques et qui ne sont peut-être que l'écume de problèmes plus profonds (RACINE, 1994). Parmi ceux-ci nous retiendrons quant à nous, et plus particulièrement en écho de la contribution d'A. CUNHA, les défis théoriques et pratiques que nous pose la Suisse urbaine à l'orée du ze millénaire, à travers un ensemble de questions qui ne concernent d'ailleurs pas seulement la géographie de la Suisse, mais aussi, plus généralement et corrélativement, la manière de pratiquer la géographie urbaine et de lire l'avenir de la civilisation urbaine. A cet égard, plutôt que de prévoir l'avenir, nous ferons état de nos hésitations.

\section{Hésitations quant à nos représentations de l'idée de ville: vers une métropolisation généralisée?}

Les villes possèdent une concentration disproportionnée de la capacité productive nationale et de ses actifs fixes, en même temps que des ressources mobiles de capital et de travail, toutes situées sur des portions relativement restreintes du territoire. Principaux réceptacles où s'est cristallisée la mémoire historique de l'humanité, elles sont les lieux clés de l'innovation et du changement technologique. Elles servent de points de contrôle du système économique et de son organisation politique en même temps qu'elles en sont la fenêtre sur le reste du monde, reliant le pays à l'économie globale. Mais elles sont aussi le moule où s'enracine l'essentiel de nos rapports biologiques et sociaux, en même temps que le miroir où se reflètent ce que nous sommes aujourd'hui. Deux facettes d'une réalité complexe et multiscalaire que la géographie se doit continuer de saisir dans leurs rapports multiples.

Le problème est que sous le choc brutal d'une croissance mal maîtrisée et faute d'une vision globale de leur destin, les hommes qui les habitent et les aiment ou, au contraire, cherchent à les fuir, en viennent à se demander si les villes ne sont pas en train de perdre la mémoire, celle d'un patrimoine millénaire, sans rien créer de mémorable pour autant, les uns et les autres commençant à prendre conscience qu'en dehors de quelques élucubrations architecturales qui ont pu amuser certains pour un temps, rien ne prouve que la ville moderne débouche sur une quelconque "post-modernité». La ville est-elle dans le "post", dans le dépassement, ou dans l'impasse du patchwork aléatoire qu'elle est devenue? Comme le remarque J. CHESNEAUX (1992), "ce terme entretiendrait une illusion de sortie de crise». Difficile, dans ces conditions, de prévoir à quelles conditions justement, nous pourrons contribuer à faire converger les tendances lourdes du passé et du présent vers des objectifs de développement territorial, à la fois sociaux, économiques et spatiaux, qui permettraient d'atteindre et gérer ce qu'en général les géographes souhaitent dans la littérature qu'ils produisent: une situation d'équilibre dynamique entre villes et régions, la valorisation des ressources locales, la maitrise des coûts de fonctionnement des systèmes urbains, le renforcement de l'autonomie, la préservation de l'environnement et la qualité de la vie?

Pour autant qu'elle reste simultanément attentive au temps et à l'espace, la géographie sait bien que dans les périodes de mutations profondes, le vieux et le neuf sont et resteront profondément mêlés, dans la réalité comme dans les représentations, et qu'il est parfois difficile de faire le partage entre les deux. Certes, après plusieurs années de fausses représentations concernant une éventuelle désurbanisation, les travaux disponibles - et particulièrement ceux que nous avons pu conduire avec A. CUNHA - font la démonstration qu'«en dépit d'une diffusion sans précédent des nouvelles technologies de mâ̂trise de la distance, l'intégration et la spécialisation croissantes de l'économie contemporaine aux différentes échelles d'analyse tendent plutôt à fixer progressivement des activités tertiaires de commandement et de gestion économique dans un petit nombre de grands centres pouvant offrir un large éventail de professions à haute qualification». Ces études témoignent donc de la pérennité de nos villes. Elles recoupent d'ailleurs les résultats déjà mis en évidence en France par D. PUMAIN et TH. ST.JU. LIEN (1988). Bonne nouvelle dirons-nous, saisie à une certaine échelle et selon l'un des angles de vision possibles. Mais il suffit de changer l'angle d'observation de quelques degrés pour devoir tout de même s'interroger sur la pertinence actuelle et future de la notion de ville ou celle de pôle de développement - telle que traditionnellement construite et pratiquement toujours rattachee à l'évocation de son centre historique, qui, de fait, n'est plus que l'un des éléments constitutifs de nos métropoles en devenir au sein desquelles les "villes» ont définitivement perdu leurs structures matérielles et formelles traditionnelles.

Même si, pour des raisons que les géographes connaissent bien ou apprendront encore à reconnaître, les modalités du développement territorial restent et resteront sans doute très variables d'une ville à l'autre et d'une région à l'autre, la recomposition du territoire autour des 
grands centres urbains et de leurs réseaux ne peut être qu'amplifiée par le processus d'intégration au système de communications européens. A très court terme, il faudra convenir que les unités de mesure ne seront plus ni les blocs ni les quartiers, mais les corridors de croissance et autres "corridors de haute technologie», s'inscrivant de plus en plus fréquemment dans les «nouveaux créneaux d'opportunité géographique» chers aux théoriciens du capitalisme flexible post-fordiste. Les indicateurs de la centralité ou de la périphéricité sont multiples, inégalement et pas toujours positivement corrélés dans l'espace. En se transformant en région urbaine, la "ville» garde peut-être quelque chose de son identité, mais il ne fait plus de doute, et pas seulement en Amérique du Nord, que les nouvelles régions urbaines tendent à être polycentrées, multicentrées, avec des centres de troisième, quatrième et même cinquième générations localisés dans et au-delà des cités de lisière et des corridors de croissance autour desquels s'étalent de grandes communautés résidentielles planifiées par la promotion privée (LEIMBERGER, 1990, KNOX, 1991; LERESCHE et BASSAND, 1991). De là l'émergence d'une représentation possible de notre devenir quotidien, qui aura au moins le mérite de stimuler une certaine recherche géographique, comme il la stimule actuellement aux Etats-Unis (BERRY, HAK-MIN KIM, 1993), lui donnant l'occasion d'heureuses "découvertes", ne serait-ce que celle de la mort de ses traditionnels gradients centres-périphéries, applicables à tout et n'importe quoi, puisqu'aussi bien d'autres nœuds que ceux des anciens CBD exerceront une grande influence, et que ce que nous avons appelé les lieux centraux, n'étant plus des lieux, et n'étant plus au centre, les périphéries n'en seront plus, du moment, rappelle A. CORBOZ (1993), que la notion même de périphérie suppose un point focal. De nouvelles courbes en U inversées refléteront même bientôt le jeu entre les externalités négatives du centre et les valeurs de la proximité. Des superpositions multiples et une multitude d'influences spatiales vont jouer les unes contre les autres impliquant l'adjacence des espaces, le voisinage, l'environnement, de nouvelles valences affectives et emblématiques sans doute aussi.

Avec l'émergence de l'urbanisation extensive, de la ville diffuse, voire "ville-territoire» ou «l'hyperville» chères à A. CORBOZ (1993), ne convient-il pas de revoir nos modèles et nos outils de travail, en vue de construire une nouvelle théorie de la dynamique territoriale? Certains s'y exercent déjà. Ainsi P. WADDEL, B.J.L. BERRY et I. HOCH (1993) dans le prolongement des études sur la New City américaine et sur la cité informationnelle (CASTELS, 1989). Nous devrons en tirer les conséquences. L'essentiel ne consiste-t-il pas dans la capacité du réseau à transférer l'information entre chaque ensemble d'activités et les localisations domestiques, cette capacité différentielle devant créer des ordres et des opportunités de travail pour les groupes sociaux les plus qualifiés et autoriser des configurations d'emploi plus flexibles avec une part plus grande de travail fondé sur la maison? De fait, soumis à des pulsions qui renvoient aux relations qui s'établissent entre l'économie et l'espace, nos espaces en cours de métropolisation généralisée connaissent des distorsions qui se traduisent par des déclassements/reclassements, délocalisations/relocalisations continus remettant en cause l'association entre centre et centralité. En regard d'une double logique, liée à la mondialisation de l'économie et imprimant à nos agglomérations leurs intérêts propres, l'une touchant à certains espaces stratégiques de l'agglomération en termes économiques, l'autre se focalisant sur quelques périmètres bien déterminés dans la ville centre, d'autre part, il n'est pas si évident qu'ils s'agisse des élus ou des habitants en général, que les populations concernées puissent s'adapter sans risque aux mutations qui se déroulent dans l'espace urbain et ce d'autant plus que ces pressions symbolisent les restructurations économiques qui dépassent de loin le cadre des forces économiques locales (WOLF, 1993).

"Cette évolution s’inscrit bien dans une logique de communication et non de communion", nous dit A. CUNHA en évoquant un scénario possible qui irait renforcer le rôle dominant d'une logique qui, pour être consubstantiellement urbaine, reste, même sous la forme de l'hyperville, la logique de la ville productiviste, informationnelle, transactionnelle, fondée sur des espaces de flux, avec ses corrélatifs spatiaux et comportementaux, branchés sur les grands réseaux et autres autoroutes de la communication. C'est le scénario moderniste de la métropolisation à outrance avec ses configurations composées de différents réseaux superposés, ayant chacun sa logique propre, rendant obsolete toute une terminologie et des représentations de l'urbain dérivant encore de la métropole industrielle. Il reste que la constitution de ces nouveaux tissus urbains n'est encore que l'expression du renforcement du rôle économique et social, à l'échelle du pays, des métropoles anciennes. C'est encore vrai au niveau structuro-fonctionnel, ça l'est surtout au niveau socio-affectif( REMY et VOYÉ, 1993), où ni la déconcentration de la population et des emplois au sein de l'hyperville ni la forte croissance de certains pôles secondaires ne doivent faire illusion.

Invariance dans le changement? La centralité urbaine est un des éléments qui structure fortement les rythmes de fréquentation et les choix de localisation, de délocalisation et de relocalisation des acteurs économiques mais aussi le système de relation des habitants à leur territoire dans un autre registre que l'économique, celui du vécu et, d'une manière plus générale, celui du culturel. A l'intérieur d'un espace multimodal, les habitants vivent eux aussi et de plus en plus une "cité à la carte». Ce qui n'empêche pas, heureusement, nos principaux centres urbains d'en demeurer (encore) le principal élément structurant par la densité de leurs liens et la dimension des effets de polarisation, par rapport à tous les centres secondaires, ou aux différentes zones fonctionnelles, qu'elles soient bleues, vertes ou grises, et ce très significativement, dans le domaine des activités ludiques et culturelles qui restent le fin mot de l'urbanité. Pour combien de temps? Et le dire ainsi, de manière implicitement normative, en cherchant à valoriser la ville comme lieu de ren- 
contre et de confrontation, de communion tout autant que de communication, n'est-ce pas se refuser à imaginer un autre modèle, où la centralité et ses attributs, plutôt que de rester enfermés dans un modèle hiérarchique cristallé rien se diffuseraient dans une structure en réseau de type réticulaire?

\section{Hésitations devant le nouveau contexte social}

D'un côté, certains se plaignent de l'étiolement et parfois de l'implosion du cœur des villes, dont témoigne par exemple le mouvement de désaffection qui gagne plusieurs grands centres, accompagné d'une substitution de population mal intégrée à une population d'esprit citadin qui, elle, part résider dans une périphérie aux limites de plus en plus floues, la ville y perdant ses structures matérielles et formelles. Discours largement entendu à Détroit ou Los Angeles et, plus près de nous, à Marseille, mais également en Suisse aussi (PIVETEAU, 1989). Force est de constater pourtant que les discours inverses ont également et légitimement droit de cité (SÖDERSTRÖM, 1994), voulant que la globalisation de l'économie et l'empreinte du capitalisme avancé conduisent actuellement à une recentralisation prenant de fait la forme complexe d'une urbanisation congestionnée, fragmentée au sein de laquelle le processus d'élitisation des centres (la gentrification des auteurs anglo-saxons) introduit de nouvelles polarisations, toujours difficiles à assumer. La gentrification a, en effet, ses corollaires obligés dont l'exclusion à terme des plus faibles et des marginaux de tous types. L'exclusion, c'est-à-dire la rupture de rapports sociaux et culturels, ceux-là même qui, justement, fondaient l'idée de ville. Ces polarisations s'inscrivent dans un phénomène plus général de partition qui renvoie lui-même à l'opposition entre exclusion et participation. La nouvelle société urbaine est de plus en plus considérée comme une "fabrique d'exclus» (TOURAINE, 1991). Même dans les pays occidentaux les plus avancés et les plus riches, le constat s’impose: la société à deux vitesses devient la composante dominante de la structuration sociale, et spatiale. Nous vivons dans un espace divisé, un puzzle complexe en fait, multidimensionnel et multiscalaire. Dont le principe, au sens premier de cause agissante mais aussi de règle de composition, semble avoir considérablement évolué ces vingt dernières années et porte aujourd'hui un nom incontournable, celui de réseau. "Etre ou ne pas être dans le réseau, telle est la question.» II y a ceux qui sont «branchés» comme on dit, et il y a les exclus, ceux dont on n'a que faire dans la nouvelle histoire qui commence avec les années 80 , celle du capitalisme post-fordisme, flexible, porteur de nouvelles forces économiques, sociales, politiques, culturelles (RACINE, 1992).

Force est de constater aujourd'hui que tend à s'imposer un peu partout la thématique de la pauvreté urbaine et métropolitaine, couplée avec l'indispensable interrogation sur les raisons qui font que des zones, des pans, des fragments de la société urbaine, au double niveau des êtres humains et des espaces, des territoires, se décompo- sent, se marginalisent, dérivent. On en vient à se poser la question de savoir si la vulnérabilité des métropoles, c'est la vulnérabilité des villes, ou plutôt celle de fragments de la ville (il peut s'agir des centres, comme à Marseille, il peut s'agir des banlieues comme à Mantes-la-Jolie ou Vaulx-en-Velin)? C'est peut-être au cœur d'une des deux ou trois villes considérées comme les plus riches du monde, avec la scène ouverte de la drogue au Letten, avec toute la criminalité qu'elle entraîne, que le problème est le plus virulent, son attrait étant devenu si important, et sa taille si gigantesque qu'elle ne peut plus être vaincue par la police et ce de l'aveu même du chef du Département de la justice zurichoise.

Le contexte géographique est en train de changer. Mais le contexte social qui lui est indissolublement lié change également. Dans ses différentes dimensions, démographiques, économiques, culturelles, politiques. Un changement qui est sans doute l'expression d'une série interreliee de transitions structurelles, dans les rapports et les hiérarchies sociales comme dans les styles de vie. Tout est devenu plus complexe sur le plan fonctionnel, et bien moins facilement catégorisable. Les questions se recoupent: outre celles de l'évolution du système productif (désindustrialisation diffuse et tertiairisation sélective), largement évoquées par A. CUNHA et J. B. RACINE à l'occasion du PNR-25 et de la thèse du premier (1993), la question de la natalité, de la décohabitation et de la taille des ménages, de la multiplication des familles monoparentales et des monogamies séquentielles, la question du vieillissement, des taux et des rapports de dépendance entre générations, actifs et non actifs, la question de l'évolution des sex-ratio et plus généralement des rapports gendrés, la question de la participation croissante des femmes à la main-d'œuvre active et payée et, partant des revenus combinés, la question de l'accès différentiel aux études supérieures, l'ensemble des questions liées à l'immigration, qui recoupent toutes les autres. Elles introduisent et introduiront d'importants changements dans les rythmes de la vie, à la maison comme dans les relations de voisinage. Composition et recomposition démographique, économique, sociale, ethnique culturelle de la population urbaine, certes, mais aussi manière de choisir et d'organiser son cadre de vie, son environnement de travail, les relations sociales et familiales. Sur toutes ces questions et la manière dont elles s'entrecroisent, la recherche va sans doute, et sans attendre, s'attaquer aux données du dernier recensement et produire sans doute des résultats statistiques passionnants qui enricheront grandement nos représentations de l'urbain et de la théorie urbaine, quitte à les remettre assez radicalement en cause. Mais sera-ce suffisant?

\section{Les enjeux de la recherche: connaître pour relier}

N'est-il pas tout aussi nécessaire, et d'urgence, de nous interroger sur la manière d'investir nos espaces urbains d'un sens dans lequel les habitants puissent se reconnaî- 
tre au point, minimal, que ces espaces deviennent enfin pour eux de véritables territoires gratifiants, supports d'une identité qui ne soit plus celle des parias de la croissance et d'une urbanité retrouvée ailleurs? Refaire des villes ce qu'elles étaient par essence, des lieux de l'échange et du dialogue, ce qu'elles doivent nécessairement être aujourd'hui pour que cet échange et ce dialogue puissent se réaliser à nouveau: des lieux de la reconnaissance des communautés, des lieux où l'on puisse développer sans exclure, et donc des villes tout à la fois plus hospitalières et plus participatives, renouant, à leur échelle, avec une démocratie authentiquement vécue (RACINE, 1993)?

Dans son Abécédaire de l'ambiguité, le généticien A. JAC. QUARD ( 1989) compare deux signes urbains, les arches de Paris. D'une part celle de l'Arc de Triomphe qui rappelle les victoires de l'Empereur, d'autre part celle de la Défense ou il n'est plus question de gloire ou de triomphe, mais de communication entre les hommes et de droit à la dignité. "LAarche de la Défense devrait nous inciter à mépriser tous les triomphes, ceux des militaires comme ceux des financiers, car ils ont tous un goût de mort. L'important est de défendre la vie; seules méritent nos efforts les arches de l'alliance."

Evoquer l'alliance évoque irrésistiblement une idée d'unité, d'unité à refaire, l'idée aussi de convivialité, de commune participation au même festin, qui elle-même renvoie à celle d'une société ouverte contre ceux qui veulent une société fermée, pour défendre l'intégration contre l'exclusion et appuyer les campagnes de lutte contre le racisme et la xénophobie. Encore faut-il s'efforcer d'aller au-delà de la simple opposition entre le bien et le mal, la vertu et le vice, en cherchant à mieux identifier les obstacles à surmonter. Une action ne peut pas être efficace si elle ne s'appuie pas sur une analyse objective de la situation, y compris de la situation telle qu'elle est subjectivement vécue par les différents intéressés, sachant bien par exemple que les difficultés rencontrées ne viennent pas pour l'essentiel des immigrés mais de la société qui les reçoit. Quelles sont ses valeurs, et quelles sont ses priorités? Elles vont jouer un rôle tout à fait considerable dans le devenir de nos villes et de nos banlieues. On ne les connait pas, comme on connait encore mal de fait, leurs conditions de vie réelle.

Nul doute qu'en dépit de l'urbanisation généralisée de nos territoires, les connaissances et les pratiques qui pourraient fonder le savoir et le pouvoir liés à une théorisation de l'urbain restent encore bien en-deça de la demande sociale. Cela est vrai dans les différentes disciplines qui touchent à l'urbain à propos desquelles bien des responsables ont l'impression que non seulement elles ne disposent plus d'un savoir et d'un savoir-faire «à date" ou conforme aux besoins actuels, mais encore qu'elles ont souvent, et littéralement, régressé. Le paradoxe étant que cette régression viendrait, pour les uns, du rejet post-soixante-huitard, de tout ce qui a un caractère technique, quantitatif ou formalisé, et qu'illustre le mouvement humaniste en géographie et, pour les autres, d'un excès de formalisation, illustré par ceux qui écrivent au- jourd'hui dans Economic Geography ou Geographical Analysis, excès partiellement responsable, entre autres, d'un ratage, au sens de manquer l'objectif, des problèmes et des enjeux véritables, dans la manière d'étudier la ville comme dans le choix des problemes pertinents. Ceux-ci ont évolué beaucoup plus vite que nos connaissances et nos pratiques, au point que certains jugent qu'en regard, nos disciplines sont de grandes muettes.

Reste à savoir quels sont les enjeux principaux de la recherche et de l'action en la matière. Avec R. PATRELLA (1992), nous retiendrons les questions suivantes:

- Qui construit la ville à l'heure actuelle? Les opérations immobilières? L'événement fantastique, les olympiades tous les quatre ans, les expositions universelles, l'exposition Van Gogh, etc...?

- Qui décide dans la ville? Qui décide aujourd'hui dans notre vie démocratique? Où est la démocratie représentative dans les tissus europeens?

- Qui vit la ville? S'agit-il de ces flux de voitures qui s'engouffrent dans la ville et l'engorgent, et qui la mettent chaque matin et chaque soir en état d'infarctus immédiat? A cet égard, le sociologue G. MARTINOTTI (1991) rappelle que pendant des siècles, les villes ont été formées par des gens qui travaillaient et habitaient au même endroit. Les villes étaient, en quelque sorte, fermées. Puis, les murs ont été détruits et on est peu à peu passés à un autre stade, avec le développement d'un cercle plus large oủ les gens habitaient mais ne travaillaient pas. C'est l'épopée de la pendularité, phénomène essentiel peut-être, du milieu de ce siecle. Or quelle est la situation aujourd'hui à l'heure où se multiplient les «Edges Cities»? De nouvelles structures métropolitaines se mettent en place, liées entre autres, aux configurations formées par les différentes destinations de ses non moins différentes populations d'utilisateurs, qui soit y résident et y travaillent, soit y travaillent sans y résider, soit se contentent de l'utiliser à certaines heures de la journée, avec pour cas limite celui des métro-businessman qui se retrouvent dans les World Trade Center qui uniformisent les skyline de toutes les villes d'une certaine importance. Dans le système que G. MARTINOTTI propose d'appeler "City Use», la ville, son cœur, deviennent de plus en plus des espaces de pure consommation de biens et de services. Une réalité encore bien peu analysée. Et demain, dans dix ans, avec la multiplication et la généralisation du téléphone cellulaire, qui vivra la ville?

C'est l'une des dimensions du problème. Il en est une autre, qui renvoie évidemment à la dialectique des inégalités sociales, de l'inclusion et de l'exclusion. Il ne s'agit évidemment plus de découvrir ces inégalités. La question est de savoir si nous sommes dans une société qui va de plus en plus spatialiser ces inégalités et qui va les rendre dans une certaine mesure irréversibles ou au contraire dans une société qui va pouvoir les traiter, en se fondant sur les valeurs de dignité et d'enracinement? On peut se demander si notre société porte en elle-même la capacité de réduire ces écarts et surtout si elle n'y pousse pas par ses propres divisions du travail, par ses propres statuts 
modifiés du travail, par son propre systeme de valeurs. Or qu'existe-il aujourd'hui comme systeme de valeurs commun?

La recherche pourra sans doute contribuer à repenser la situation, mais la vraie question est bien de savoir ce que devient notre idéal démocratique, qui suppose une «égale dignité de tous»? Nous sommes convaincus qu'il nous faudra réfléchir collectivement à la (meilleure) manière pour le secteur public de s'ajuster a la demande croissante pour de nouveaux types de biens publics, demande provenant d'une population non seulement plus importante mais de plus en plus diverse et discriminatrice en termes de besoins, dans des cadres institutionnels qui seront nécessairement à réviser, et probablement à inventer. A l'heure ou nos territorialités urbaines s'inscrivent dans des territorialités à la carte, au niveau micro-local, une politique urbaine globale est de plus en plus nécessaire. Quelle que puisse être notre culture fédéraliste, il est difficile de la concevoir comme étant du seul ressort des structures politiques cantonales.

Cette manière de voir a certainement pour effet de limiter la portée et l'efficacité des mesures prises ou envisagées pour assurer la gestion et le developpement des villes, ces structures politiques correspondant de moins en moins à l'espace vécu. La création d'un Secrétariat d'Etat aux villes ne pourrait-elle pas stimuler la reconnaissance d'un plus grand pouvoir aux villes, pour autant qu'il s'inscrive dans une réalité nouvelle, née de la fusion, de l'intégration ou de toute autre forme contractuelle efficace et démocratique, dans la "ville» ou l'«hyperville» des communes dites de banlieues, urbaines ou suburbaines, expression territoriale du mouvement d'extension des villes de la période 1945-1975? Nombreux sont ceux qui le pensent en Suisse romande, autour de la Syndique de Lausanne, Mme Yvette Jaggi, et, peut-être, du moins l'espèrent-ils, autour de la Conseillère fédérale Mme Ruth Dreifuss. Les marges de manœuvre pour une politique volontariste sont si faibles face aux tendances lourdes de la métropolisation que tous les efforts seront les bienvenus, pour que toute l'attention soit accordée aux clignotants qui s'allument, et que si révolution culturelle il y a, celle-ci trouve tout de même dans nos édiles l'indispensable oreille attentive, en même temps que les moyens d'agir.

\section{Bibliographie}

BASSAND, M., JOYE, D., SCHULER, M. (1988): L'urbanisation de la Suisse: faits et perspectives. Dans: Les enjeux de l'urbanisation, OEPR, Peter Lang, Bern, p. 51-69.

BERRY, B. J. L. (1973): The Human Consequences of Urbanisation. London and Basingstoke, Macmillan.
BERRY, B.J.L., et HAK-MIN, K. (1993): Challenges to the Monocentric Model. Dans: Geographical Analysis, 25, 1, 1-4. CASTELLS, M. (1989): The Informational City, Oxford, Basil Blackwell.

CHESNEAUX, J. (1992): Insoutenable modernité. Dans: La Revue Nouvelle, 23, XCV, 54-63.

CORBOZ, A. (1987): Vers la Ville-Territoire. Dans: Actes du colloque scientifique à l'occasion de l'inaguration du bâtiment complémentaire de la Haute Ecole Commerciale de St-Gall, Berne et Stuttgart, Paul Haupt (éd.).

CORBOZ, A. (1993): La Suisse, fragment de la galaxie urbaine européenne. Dans: Revue Economique et Sociale, 51 , 4. 253-260

DOLLE, J.P. (1990): Fureurs de ville. Paris, Grasset.

FISHMAN, R. (1990): America's New City. Dans: The Wilson Quarterly, 14, 24-48

JACQUARD, A. (1989): Abécedaire de l'ambiguité, Paris, Seuil, coll. Point Virgule.

LAMBELET, J. C. (1994): Bien-être matériel. Dans: Bilan, 2 , p. 27.

MARTINOTTI, G. (1991): The New Metropolis: social morphology and governance. Dans: Future of European Cities, European Research Conference 1991 Series. Espinho.

PIVETEAU, J. L. (1986): Le fait urbain en Suisse depuis le milieu du XIXe siècle: Rétrospective et vues contemporaines successives. Dans: CLAVAL, P. (éd.): Gégraphie historique des Villes occidentales, tome II, Paris, Publications du Departement de Géographie de I'Université de Paris-Sorbonne, no 14

PROGNOS (1994): Pays industrialises 1992-1998-2005. Bâle, Centre européen d'études économiques appliquées.

RACINE, J.-B., CUNHA, A. (1990): Invariance and structural changes in the Swiss urban System: the leading role of tertiary activities. Dans: BOURNE, L. S., et al:: The Changing Geography of urban Systems, Perspectives on the Developped and developing Worlds, I. G. U. Uni. de Navarra, Dept. of Human Geography, p. 145-172.

RACINE, J.-B., CUNHA, A. (1991): La recherche urbaine en Suisse, ou comment avoir le beurre et l'argent du beurre. Dans: PUMAIN, D., SAINT-JULIEN, T., et VAN DER HAEGEN, H.: Cities in Movement, Urban Systems on the Eve of the $21 \mathrm{st}$ Century, Acta Geographica Lovaniensia 31, p. 164-172.

RACINE, J.-B. (1992): Développement urbain métropolitain et crise de la ville: un monde en transition. Dans: Revue suisse d'économie politique et de statistique, vol. 128(3), 241-260.

RACINE, J.-B. (1993): La ville entre Dieu et les hommes, Paris, Anthropos et Lausanne, Presses bibliques universitaires.

RACINE, J.-B. (1994): Langues et identités territoriales en Suisse: les leçons géographiques d'un vote historique ou la Suisse à l'heure du rideau de rösti. Dans: Annales de Géographie, vol. 2 (sous presse).

REMY, J., et VOYE, L. (1992): La ville: vers une nouvelle définition? Pairs, L'Harmattan.

ST-JULIEN, T., PUMAIN, D. (1989): A ville plus grande, travail plus qualifié. Dans: Les Annales de la Recherche urbaine, no 29, p. 105-118.

TOURAINE, A. (1991): Face à l'exclusion. Dans: BAUDRILLARD, J., LIPOVETSKY, G., et al.: Citoyenneté et urbanité, Paris, Esprit.

WADDELL, P., BERRY, B.J. L., et HOCH, I. (1993): Housing Price Gradients: The intersection of Space and Built Form. Dans: Geographical Analysis, 25-1-5-19. 